\title{
La simulación social, ¿̨una nueva manera de investigar en ciencia social?
}

\section{Carlos Lozares}

Universitat Autònoma de Barcelona. Departament de Sociologia

08193 Bellaterra (Barcelona). Spain

Carlos.lozares@uab.es

\section{Resumen}

Este artículo ofrece un sucinto panorama de la simulación social, presentándola como un medio para modelar los procesos sociales y como un instrumento para la investigación social. En la introducción, se definen conceptos clave como modelo, modelo matemático, simulación social e inteligencia artificial social. En la segunda parte, se muestra la extensa variedad de modelos de inteligencia artificial social y sus aplicaciones, tanto a partir de la simulación clásica como en sus nuevas manifestaciones. Finalmente, se brindan algunas reflexiones de carácter más metodológico sobre la simulación social.

Palabras clave: modelo, modelo matemático, inteligencia artificial, sistema experto, nueva inteligencia artificial, simulación social.

\section{Abstract. Social simulation: a new way to do research in social science?}

This papers offers a brief review of the social simulation, which can he considered a way to model social processes, and also as an instrument for sociological research. First of all, key concepts such that model, mathematical model, social simulation and social artificial intelligence are defined. Secondly, a variety of models of social artificial intelligence and its applications are displayed. Finally, there are some methodological consideration about social simulation.

Key words: Model, mathematical model, artificial intelligence, expert sistem, new artificial intelligence, social simulation.

\section{Sumario}

Introducción: modelos, modelos matemáticos en sociología, simulación social e inteligencia artificial

1. Los modelos matemáticos y los de simulación
2. Los procedimientos de simulación o tipos de inteligencia artificial (IA) y la inteligencia artificial social (IAS)

3. La simulación, la sociedad artificial y la sociedad real

Bibliografía 


\section{Introducción: modelos, modelos matemáticos en sociología, simulación social e inteligencia artificial}

Un modelo es la representación que bajo ciertas condiciones se tiene o se atribuye a un sistema real o formal. Dicha representación es susceptible de expresarse de varias formas, por ejemplo: por el lenguaje natural, por concreciones físicas como maquetas (modelos reducidos o analógicos), por gráficos y también por los lenguajes matemáticos (y/o lógicos) y computacionales. Dado que un modelo puede tener diferentes modos de expresión, modelar un hecho o una entidad mental no es en principio incompatible con ninguna opción metodológica dentro de las que se dan en sociología: los tipos ideales de Weber no son mas que una manera de modelar la realidad social. En este sentido, dentro de una perspectiva cualitativa se puede hablar también, por ejemplo, de modelos discursivos como representación condicionada del significado de un texto, entrevista, etc.

Los modelos pueden expresarse también en el lenguaje abstracto de las matemáticas y/o en el computacional o en modos gráficos equivalentes; la hegemonía que ha tomado el lenguaje matemático ha hecho casi equiparable la modelación genérica con la matemática. Un modelo matemático es, pues, una forma matemática de expresar declaraciones y/o proposiciones sustantivas de hechos o de contenidos simbólicos: están implicadas variables, parámetros, entidades y relaciones entre variables y/o entidades u operaciones. El éxito de la modelación matemática está en encontrar la formulación que sea apropiada a la realidad que se estudia (Saaty y Joyce, 1981). Tratándose de fenómenos o teorías sociológicas tendríamos el gráfico 1 .

Expresado de un modo más abstracto, un modelo matemático es un conjunto de características que posee en común una familia de sistemas homomórficos, en nuestro caso el sistema de la realidad o de la teoría social y el sistema matemático, con un código que permite el paso de un sistema a otro. Dicho sistema de familias contiene, o se pueden inferir del mismo, las propiedades análogas que definen el modelo (Walliser, 1977).

El tetraedro del gráfico 2 lo expresa. En el vértice más alto se encuentra la idea de modelo y como base el famoso «triángulo de oro» constituido en sus vértices por la teoría sociológica, la información empírica o datos y el lenguaje matemático que nos ilustra sobre los diferentes tipos de modelos (Leik, Meeker,

Mundo social

(de los fenómenos o de las teorías sociales)

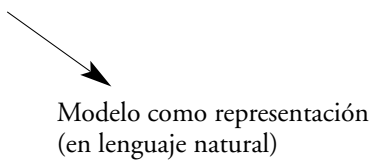

(en lenguaje natural)
Matemáticas

(álgebra, estadística...)

Gráfico 1. 


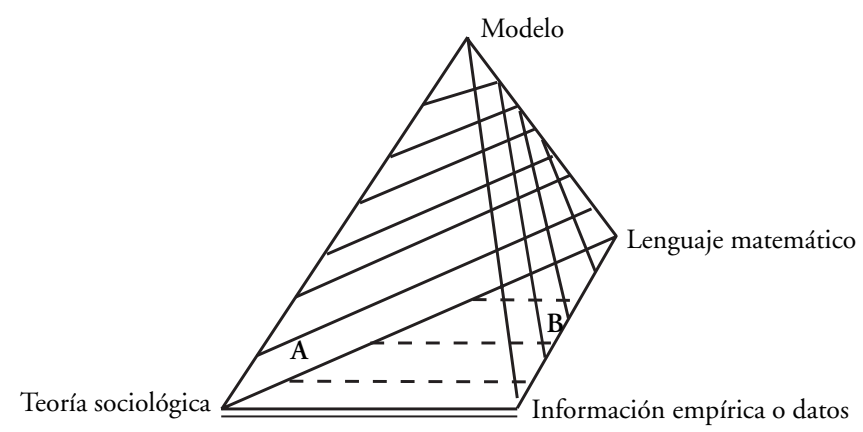

Gráfico 2.

1975): un modelo matemático más formal versaría hacia la cara A del tetraedro y uno más metodológico, hacia la cara $\mathrm{B}$.

Los modelos matemáticos usados en sociología, como en las ciencias sociales, tienen diferentes funciones, objetivos, lenguajes y temáticas, de ahí su diversidad. Los roles del modelo van desde formalizar matemáticamente teorías sociales fundamentadas axiomáticamente, como ha sido el ideal de muchos sociólogos matemáticos, hasta trabajar con modelos metodológicos más aptos para su validación. Los modelos pueden ser exploratorios o heurísticos que posibilitan la generación conceptual y tipológica o una mayor precisión del mismo modelo; descriptivos; predictivos que proveen datos proyectados en estados no observados y/o futuros del sistema; explicativos que permiten la elucidación de los mecanismos y procesos «esenciales» de los fenómenos sociales. Según el lenguaje matemático usado se puede tratar de modelos estocásticos o deterministas, de ecuaciones lineales, logísticas, estadísticos, matriciales y grafos, reticulares, etc. A su vez, se pueden modelar diferentes contenidos o procesos: la emergencia, caos y catástrofes, la estructura social, la movilidad y los cambios, la difusión y causalidad, etc. (Seror, 1994; Gross y Strand, 2000). En consecuencia, conviene tener clara la distinción entre modelo en sí o genérico y su especificación en un lenguaje concreto que determinará no sólo su forma, sino también la lógica de su funcionamiento, por ejemplo, un conjunto de ecuaciones matemáticas o un programa de ordenador son especificaciones (Doran y Gilbert, 1994).

En las últimas décadas se ha mostrado que muchos de los problemas (demostrar teoremas, reconocer pautas de comportamiento, tratar situaciones complejas, operar con la probabilidad, etc.) que solamente eran tratables y resueltos por las capacidades humanas como las de pensar, razonar, memorizar, aprender, observar, percibir, modelar matemáticamente la realidad etc., también han sido tratados y resueltos por las máquinas o artefactos $\mathrm{y}$, concretamente, por ordenadores. La inteligencia artificial (IA) puede definirse como un sistema computacional con capacidades y/o características que producen resultados 
comparables o iguales a los resultados de la inteligencia y/o acción humana, y ello no tanto porque imiten o copien el comportamiento humano, sino porque son equiparables en sus consecuencias o resultados. El campo de la IA es muy amplio, el de los sistemas expertos (SE), los dinámicos y evolucionarios, la interacción entre agentes, la percepción y reconocimiento del habla natural, de las imágenes y pautas, la simulación de la teoría, del aprendizaje, etc. Los $\mathrm{SE}$, que es al que más genéricamente nos referimos cuando hablamos de IA, no son más que una parte, importante sí, de un movimiento mucho más generalizado y amplio de la IA (Castillo, Gutiérrez y Hadi, 1997). La simulación por computación, como resultado prominente de la IA, se ha afianzado como una metodología útil para modelar fenómenos de interés científico social, por ejemplo, los de cooperación, de coordinación, de dinámica social, de formación de grupos y coaliciones, los sistemas de acuerdos y normas y su evolución, los comportamientos normativos, la generación y el desarrollo de instituciones y organizaciones, el estudio de la actividad y agentes situados, los procesos adaptativos, inteligentes, flexibles y evolucionarios, la coordinación entre agentes que interactúan automáticamente, la emergencia de los fenómenos sociales, la complejidad de las redes sociales, etc.

La vinculación entre sociología y simulación por ordenador es un fenómeno reciente, creciente y seguramente imparable. Si la sociología se queda fuera de este tren en marcha, primero, perderá oportunidades de avanzar teórica, metodológica y empíricamente, en el tratamiento de los datos y, segundo, otras disciplinas lo harán en su nombre, usando o no los adquiridos disciplinares acumulados por la sociología, como ya sucede de hecho actualmente. Parece pues necesario que los sociólogos nos acerquemos a (y nos formemos en) los procedimientos, modelos y métodos de simulación, formación que no consiste necesariamente en saber programar, sino en saber expresar, leer e interpretar lo social y su conocimiento (los agentes y actores, las estructuras, acciones, equilibrios, etc.) en mundos artificiales con modelos, diseños, experimentos y resultados virtuales y con los lenguajes de los programas de ordenador (Carley, 1996). Veamos más de cerca las diferencias entre la modelación matemática y la modelación por simulación.

\section{Los modelos matemáticos y los de simulación}

En ese apartado trato de poner de manifiesto, a través de la comparación mutua, las diferencias que se dan entre la utilización de los modelos de simulación y los matemáticos. Se sostiene que los modelos de simulación suplen o resuelven algunas de las carencias o dificultades que tienen los modelos matemáticos en su aplicación a la sociología.

El gráfico 3 representa un modelo de simulación, es decir, la reproducción artificial de los fenómenos sociales. Intervienen en ello todos los elementos del gráfico.

El sistema social modelado puede ser el de una realidad social, como es el caso de un conjunto de actores individuales (o grupos) que interactúan según 


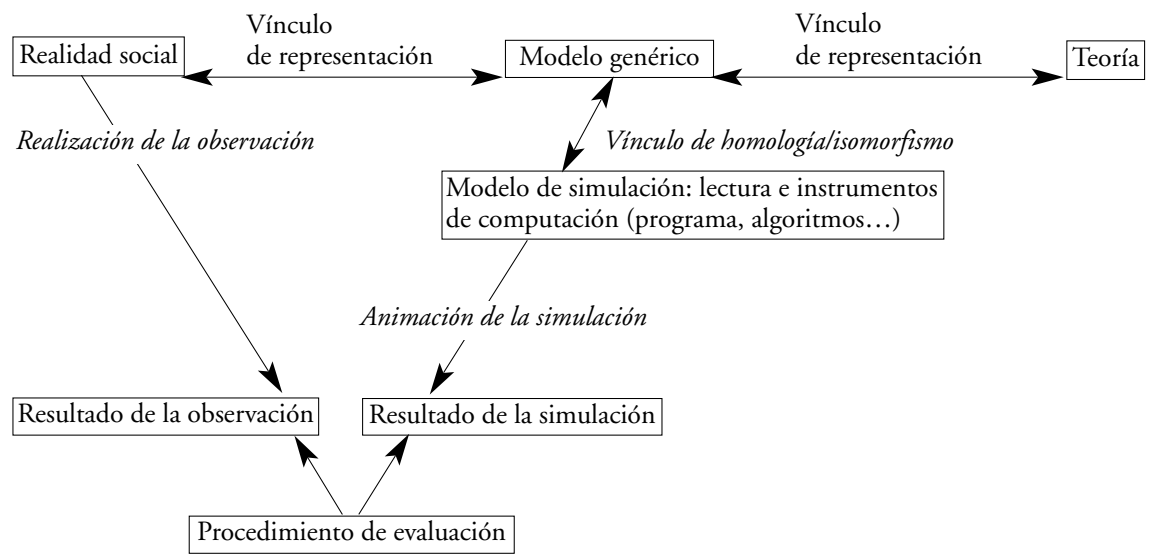

Gráfico 3 .

pautas adaptándose a las contingencias del entorno, pero puede ser también un sistema teórico social. El modelo genérico es una representación, una imagen o una definición general y/o abstracta del sistema social, tal y como se ha definido precedentemente. El lazo o vínculo de esta representación es una función de analogía, semejanza, aplicación u homología o, quizás, hasta de isomorfismo que proyecta los elementos, las propiedades y las relaciones del sistema social en el modelo. El modelo de simulación computacional es la expresión del modelo genérico en el lenguaje de ordenador, el lenguaje puede ser numérico o simbólico. Lo que parece claro es que debe darse un grado de homología o equivalencia entre el modelo de simulación específico y el genérico. La simulación consiste, pues, en el hecho de la animación o ejecución de un programa (de simulación) de ordenador en vistas a la producción de resultados en un mundo social artificial según la lógica del modelo que representa el programa. Los datos que se simulan provienen de datos observados o de datos aleatorios o, incluso, de datos simulados (Doran y Gilbert, 1994).

Los datos observados se obtienen en y del mundo real por procedimientos de recogida de datos. La evaluación o comparación entre datos esperados de la simulación y los observados se lleva a cabo por una metodología adecuada. Los modelos de simulación social, como cualquier modelo, poseen grados de agregación, esto es, pueden referirse a la totalidad del objeto estudiado o a una de sus partes, a veces gran parte de su importancia radica en ello.

Los modelos matemáticos tienen ventajas indudables sobre los modelos verbales y/o del lenguaje natural: mayor precisión y claridad conceptual y de los supuestos, mayor posibilidad y capacidad para decidir sobre la validez interna y externa de las hipótesis así como un mayor poder de deducción formal y, por fin, mayor facilidad de comunicación sin ambigüedad entre científicos 
debido al lenguaje formal. Han sido y seguirán siendo utilizados a pesar de los vituperios recibidos por algunas corrientes metodológicas de sociología, ya que han procurado resultados eficaces e interesantes para el progreso de la sociología, sobre todo los llamados «modelos matemático-metodológicos», más prestos para su validación que los modelos matemáticos formalizados de teorías axiomáticas. Pero los modelos matemáticos cuentan también con limitaciones importantes por su grado de simplificación y rigidez (Taber y Timpone, 1996). Eso no es óbice para que puedan vislumbrarse, como sucede de hecho, otros modelos matemáticos más flexibles a partir de otros tipos de álgebras diferentes de la lineal.

Como he afirmado, los modelos matemáticos consisten en un conjunto de expresiones algebraicas y/o estadísticas que son representaciones abstractas de los procesos sociales estudiados. El funcionamiento del modelo matemático es el siguiente. En un primer paso, se calculan los parámetros de las ecuaciones del modelo, estimación que se lleva a cabo a partir de los datos observados por medio de procedimientos estadísticos. A partir de dichas ecuaciones, se generan predicciones de los componentes o variables endógenas del sistema de ecuaciones que se validan por tests de ajuste, comparando dichas predicciones «esperadas» con los valores observados.

El modelo de simulación se expresa en el lenguaje de un programa de ordenador y la generación de valores esperados bajo dicho modelo se realiza ejecutando el programa. Se evalúa la adecuación de modelo comparando dichos valores esperados con los observados por tests de evaluación que acostumbran a incorporarse al mismo programa de simulación.

Expresado así, parece que ambos modelos son similares; además, ambos pueden ser usados para fines de exploración, descripción, explicación o predicción, enfatizando en cada caso uno u otro de los objetivos. Pero hay diferencias notables entre los modelos matemáticos y los de simulación. Las representaciones de los procesos que conforman los modelos de simulación acostumbran a ser más similares a los procesos del mundo real. Los modelos matemáticos son representaciones abstractas, pues están mediadas, primero, por las variables que son, de por sí, un nivel inicial importante de abstracción matemático-conceptual y, segundo, por las correlaciones, asociaciones o distancias algebraico-estadísticas que son, a su vez, abstracciones de segundo nivel sobre la relación y las pautas de comportamientos de la realidad. Raramente el modelo matemático tiene una similitud descriptiva con los mecanismos que subyacen a las relaciones que se dan en el mundo real social (Gilbert y Troitzsch, 1998).

Podemos además encontrar razones por las que la simulación puede ser más apropiada para la representación de los hechos (o teorías) que los modelos del lenguaje natural e, incluso, matemáticos y, por tanto, más eficaces e informativos:

a) Como se acaba de afirmar, los lenguajes de programación por simulación suelen ser más expresivos, próximos y cercanos a la realidad que represen- 
tan que los abstracto-matemáticos y por ello más accesibles, flexibles y de más fácil construcción y evaluación para los no especialistas. Pero también es cuestión de la explicación: implementar un modelo por ordenador requiere un nivel de detalle en la imagen de la estructura y el funcionamiento (explicación por mecanismos) que no se requiere para los modelos en lenguaje natural o en el lenguaje matemático.

b) La simulación por ordenador, especialmente si se trata de fenómenos no lineales y complejos, puede producir comportamientos no esperados, ya que pueden originar interacciones no previstas entre componentes del modelo, lo que permite la emergencia de nuevas ideas, experimentos e hipótesis.

c) Los modelos de simulación son más aptos para sistemas sociales que los matemáticos, sobre todo si la realidad social que simulan:

1) contiene dinámica, interacción e interdependencia tanto interna como con el exterior del sistema social considerado, por ejemplo, con agentes sociales heterogéneos que, además de interactuar con diferentes orientaciones sobre el mundo social, poseen diferentes stocks y capacidades cognitivas;

2) posee mayores exigencias en cuanto a cantidad y complejidad de datos;

3) supone tratamientos y/o perspectivas interdisciplinares y multidisciplinares (Seror, 1994).

d) La simulación social parece más «apta» para el tratamiento de lo social, dado que se adapta mejor a procesos que, como los sociales, suelen operan en interacciones simultáneas y sin un orden predeterminado (el orden predeterminado es más propio de los sistemas de ecuaciones). Esta mejor «acomodación» es debido a que, en general, la simulación acepta mejor una lógica de funcionamiento en paralelo y que, al ser modular, se pueden hacer cambios en una de las partes del programa sin necesidad de cambiar otras. Los modelos matemáticos, al tratar un supuesto detrás de otro, dificultan el tratamiento de las interacciones complejas.

e) Además, los modelos de simulación tienen también más adecuación y pertinencia que los matemáticos para tratar los fenómenos de complejidad, emergencia, incertidumbre, multinivel y contrafactuales, contextuales, de difusión, no lineales, inestables..., donde el tratamiento de los niveles intermedios y de los mecanismos de los fenómenos sociales son decisivos.

f) Otra dificultad de la modelación matemática clásica proviene de las escalas temporales en que se toman los acontecimientos para los que no hay datos observados: a veces, las escalas o temporalidades son más largas que la vida, con la dificultad añadida de someter los datos a un experimento. La simulación social puede abrir caminos para obviar dicha dificultad (Gershenson, 2002).

Por tanto, la simulación puede ayudar a comprender mejor por qué y cómo suceden los fenómenos sociales. Cuando se trabaja con sujetos sociales, especialmente en fenómenos interactivos, construimos modelos basados en pautas 
de comportamientos observados y los expresamos por relaciones abstractas entre variables, pero raramente podemos percatarnos de los mecanismos y procedimientos que operan y cómo operan (por ejemplo: los procesos intencionales los deducimos de una manera inferencial sin tratar de modelar sus mecanismos de actuación). Las simulaciones por ordenador permiten una mejor semejanza y un seguimiento directo. No siempre la simulación es un espejo o reflejo del comportamiento social de los sujetos, pero puede darse una estrecha correspondencia entre los comportamientos dados por los modelos y los que llevan a cabo los sujetos o agentes sociales (Plunkett y Elman, 1997; Elman y otros, 1998).

La simulación consiste básicamente en generar mundos sociales artificiales con la capacidad de producir resultados similares a los del mundo social real, lo que permite su control, reciclaje, cambio de parámetros, etc., lo que no es propio de la modelación matemática. Sin embargo, la simulación no está exenta de los riesgos de la simplificación, pues necesariamente todo modelo es un esquema para representar los fenómenos y procesos sociales (Gershenson, 2002).

El uso de la simulación por ordenador de los fenómenos sociales es relativamente novedoso pero de creciente implantación. Se inicia a mediados de los ochenta aunque hay casos puntuales en décadas anteriores. En los noventa, su aplicación a los fenómenos sociales se extiende considerablemente. Hoy día, aunque algo marginal en sociología sobre todo a causa de los sociólogos, la simulación social comienza a ser un poderoso medio de explicación y comprensión de los procesos sociales, sobre todo para otras ciencias sociales. La novedad, la extensa variedad de procedimientos y tecnologías de simulación y su vigorosa expansión, aconseja que su introducción en la sociología vaya acompañada de la exigencia conceptual y metodológica que ya posee la sociología. La aplicación de la simulación a lo social no puede reducirse, como a veces sucede con los programas de análisis matemático, a un ejercicio puramente pragmático o exclusivamente exploratorio, por no decir tecnocrático.

Algunas temáticas «sociológicas» son más recalcitrantes que otras a su tratamiento con modelos clásicos, la simulación social puede ser un mejor instrumento, por ejemplo, en la exploración, la consistencia y la coherencia de las teorías sociales (Seror, 1994); los fenómenos de emergencia social (Gilbert y Troitzsch, 1998); los sistemas sociodinámicos y/o sistemas no lineales (Helbing, 1985; Molenaar, 1994; Griffiths y Oldknow, 1993; Thom, 1975; Brown, 1995; Molenaar, 1994); el campo de la «socio-mecánica estadística» (Weidlich y Haag, 1988; Helbing, 1994); los fenómenos sociales de saturación; la teoría de juegos en la elección racional (Croon y Van de Vijver, 1994; Shubik, 1991; Helbing, 1985); la interacción (los modelos de la inteligencia artificial distribuida, de sistemas basados en agentes y los de la nueva inteligencia artificial se acomodan bien a la interacción entre agentes sociales) (Gilbert y Troitzsch, 1998); las aproximaciones reticulares; la entropía y la teoría de la información para tratar los problemas de equilibrio, estructuración, agregación y desagregación y asociación de sistemas; los modelos de análisis multinivel (DiPrete y Forristal, 1994). 


\section{Los procedimientos de simulación o tipos de inteligencia artificial (IA) y la inteligencia artifical social (IAS)}

Los primeros pasos de la aplicación de la IA a la realidad social se dan en la década de 1960 con la introducción de los ordenadores en la investigación universitaria. Las modestas experiencias de los años setenta se refieren, por ejemplo, a modelar el orden y el paso de las personas en las colas, los tiempos de espera de los coches de la policía para atender una emergencia y se centran más en la predicción que en la explicación.

Los modelos de simulación social tienen su origen en la simulación de los fenómenos físicos, biológicos y cognitivos. Veamos muy brevemente algunos de los que han tenido un origen en la física, para centrarnos luego en los de la ciencia cognitiva. La simulación dinámica se encuentra entre los primeros y más populares modelos de simulación por ordenador en ciencias sociales aplicados, por ejemplo, a los sistemas urbanos, a la población electoral (Forrester, 1969): usan las ecuaciones diferenciales (o de diferencia), para dar cuenta del estado del sistema y de las trayectorias de las variables en el tiempo. La forma de análisis es similar a la de un experimento controlado pero ahora manipulando directamente un sistema artificial (Taber y Timpone, 1996). La microsimulación es otro de los enfoques que más se propagó en las décadas de los ochenta y mitad de los noventa. En la microsimulación se atribuye una probabilidad de transición de estado a cada unidad de una muestra de la población (individuos, hogares o empresas, etc.), por ejemplo, la probabilidad de que una mujer a una edad dada tenga un hijo en un tiempo dado. El proceso se repite para el año siguiente y se va infiriendo un modelo de transición. La microsimulación ha tenido repercusión en decisiones políticas: cálculo de pensiones, de presupuestos educativos, etc. ${ }^{1}$. El automáta celular $(A C)$ se basa en los trabajos de físicos y matemáticos sobre las propiedades de la agregación de unidades (materiales magnéticos, turbulencias de líquidos, crecimiento de cristales, erosión solar, etc.). Básicamente se trata de la simulación de las propiedades de un sistema global a partir de las interacciones entre las unidades componentes, como la formación de barrios segregados étnicamente (Gilbert y Troitzsch, 1998) ${ }^{2}$.

Contrariamente a lo que sucedió en ciencias naturales, los ochenta no fueron fructíferos para la simulación social aparte de la microsimulación. Todo cambia en los noventa, con la introducción de los sistemas multiagente que posibilitan la simulación de individuos autónomos y sus interacciones con

1. La microsimulación no tiene pretensiones explicativas, sólo trata de predecir distribuciones futuras. No se consideran las interacciones entre unidades ni las intenciones entre ellas, la respuesta depende sólo de la razón de transición.

2. Un AC está definido por el número de células en una parrilla, los posibles estados de las células, la función que define las células próximas y la de transición al nuevo estado basado en los de las células vecinas. Una célula puede cambiar de estado cada vez que tres de sus cuatro laterales inmediatas están en otro diferente al suyo. Es un modelo particularmente flexible basado en la metáfora de la interacción y la reproducción celular simple. 
modelos provenientes de las dinámicas no lineal y de la IA (inteligencia artificial). El desarrollo de la simulación social en tanto que inteligencia artificial social (IAS) se fundamenta en dos formas o tipos de IA, en la inteligencia artificial de los sistemas expertos (SE) o inteligencia artificial clásica (IAC) y en la nueva inteligencia artificial (NIA).

\section{La IAS de los $S E$}

Un sistema experto (SE) es un programa de ordenador que consigue resultados comparables a los de los expertos humanos. Dado que muchos otros soportes también razonan, toman decisiones, analizan y calculan, la denominación de SE se reserva exclusivamente a modelos dentro del campo de la IA que se caracterizan porque sus procesos y representaciones al realizar tareas como inferencias, elección, control, búsqueda heurística, etc., son simbólicos. Aunque los SE son sistemas «basados en el conocimiento" se diferencian también de otras ramas de la IA por su énfasis en el conocimiento de un dominio específico más que en estrategias de resolución de problemas generales. Reflejan, por tanto, un conocimiento especializado de las tareas (Benfer, Brent y Furbee, 1991). Por tanto, los SE son programas de ordenador que proporcionan resultados equivalentes a los que produce el pensamiento o razonamiento de un experto en un área especializada. Manejan información y la manipulan para obtener resultados que sean respuestas significativas a preguntas completamente especificadas, memorizan y razonan, se comunican con los humanos, deciden y explican sus razones (Castillo, Gutiérrez y Hadi, 1997). Algunos científicos sociales consideran que los SE son modelos tan importantes para los datos cualitativos como lo fueron los paquetes estadísticos para los cuantitativos. Desde el punto de vista sociológico, lo importante es que los SE pueden representar y codificar normas, creencias, deseos o preferencias y actitudes de los expertos en un dominio social (Carley, 1996).

En las primeras investigaciones, antes de 1975, se planteaba sobre todo la formalización de conocimientos con objetivos teóricos. A partir de 1975 la simulación por ordenador comienza a emular las capacidades cognitivas y la toma de decisión de las personas con la idea subyacente de que el éxito de la simulación depende menos de la sofisticada arquitectura que procesa la información que de los conocimientos especializados y expertos que se manejan. En esta década de la ingeniería del conocimiento, los SE tienen como objetivo ayudar a la gente, sin conocimiento especialista en un campo, a tomar decisiones como si fueran expertos en él. Los años ochenta representan la segunda generación de los SE. El esfuerzo se amplía para llenar las limitaciones precedentes y extender las funciones y capacidades de la tecnología, como es el caso de las técnicas de representación del conocimiento y de aprendizaje-máquina, en los razonamientos basados en casos y explicaciones, en las respuestas a preguntas y en el razonamiento del usuario en lenguaje natural. La década de los noventa es la de la ingeniería sistemática del conocimiento. Los primeros SE fueron de pequeña escala y nacieron en los laboratorios de ciencia cognitiva y no en la indus- 
tria del software; las dos primeras generaciones de sistemas expertos no tuvieron una dimensión industrial como ha tenido en los noventa (Fox, 1996) ${ }^{3}$.

El conocimiento experto de los SE ha de ser tal que la información se almacene y se recupere fácilmente, represente completamente el fenómeno del campo especializado y que dicha información pueda ser manipulada de manera significativa, válida y sustantiva. Es lo que se llama la «representación del problema» y constituye la cuestión central de la IA (Dereck, 1996). Los SE utilizan diversos procedimientos para representar el conocimiento:

1) Los sistemas de reglas procedurales o sistemas de producción basados en el conocimiento o lógica proposicional (no, y, o, si..., entonces). En ellos el proceso de conocimiento se representa por una serie de reglas de producción, por ejemplo: enunciados reglas del tipo «si $x$ entonces $y$ ». Es decir, cuando se cumplen ciertas condiciones antecedentes se desencadena una regla que llena un consecuente que es verdadero. Las reglas están «depositadas» en el conocimiento base (Benfer, Brent y Furbee, 1991).

2) Los sistemas basados en la lógica de predicados (cuantificadores existenciales $o$ particularizados y cuantificadores universales o generalizados que cuantifican variables). En ellos el conocimiento se representa en la forma de aseveraciones, por ejemplo, la relación $R(a, b)$ afirma que $a$ tiene alguna «relación» con $b$, por ejemplo, $R$ (Juan, sociólogo) afirma que Juan es sociólogo. Los elementos pueden ser constantes o variables ${ }^{4}$.

3) Los sistemas basados en marcos son estructuras de datos que describen situaciones estereotipadas 5 .

4) Las redes semánticas son sentencias que se generalizan en redes asociativas que pueden incluir relaciones causales entre las variables. Carley (1988) usa mapas reticulares para que los programas SE codifiquen datos textuales como redes semánticas ${ }^{6}$.

3. En 1994 se habían censado ya 2.500 SE: negocios, manufactura, medicina, ingeniería, ciencias aplicadas, militar, espacio, transporte, educación, transacciones bancarias, control de tráfico, problemas de horarios, programas, planificación, optimización de objetivos, diagnóstico médico, agentes secretos (Brent, 1996).

4. Los sistemas lógicos como PROLOG usan un método de inferencia que es una estrategia para determinar si un hecho particular es válido según el conocimiento base. La lógica predicativa fue usada por Brent, Glazier y otros (1989) en la primera versión de un programa creado para razonar sobre y según Goffman.

5. Cada marco consiste en diversos trozos, slots, que representan un tipo específico de información. Los marcos, frames, se han usado para representar el conocimiento en programas de comprensión del lenguaje natural y en sociología para representar el razonamiento cualitativo de Goffman (Brent, Glazier y otros, 1989; Brent, 1996). El sistema de marcos se basa en las propiedades asociativas del conocimiento como agrupaciones cohesivas de rasgos relacionados entre sí.

6. Una red semántica define un concepto a través de un grafo dirigido que expresa relaciones con otros conceptos y propiedades. Las relaciones correspondientes son genéricas, como "es...», "tiene... propiedades», "subconjunto... de», "y... además». Pueden expresar interrelaciones y distinciones semánticas complejas entre conceptos de teorías sociológicas. Son 
5) Los razonamientos basados en casos y en representaciones analógicas. Se trata de «bibliotecas de casos sucedidos» que encuentran alguna similitud con el caso-problema real que se analiza, ofreciendo incluso estrategias para modificar la solución propuesta cuando el caso no se parece perfectamente al del problema (Dereck, 1996).

Independientemente de la estrategia de conocimiento elegida, hay dos consideraciones relevantes para los sistemas de conocimiento. Primera, muchos problemas de las ciencias sociales requieren razonar con incertidumbre. Los sistemas basados en reglas pueden acomodarse a la probabilidad introduciendo y especificando lo que se llaman "factores de confianza por medios heurísticos» (Fox, 1996). Segunda, fuera de su dominio específico, los SE no son útiles. En sociología, por ejemplo, se pueden encontrar áreas posibles para el conocimiento experto, pero se dan regiones vacías para dicho conocimiento ${ }^{7}$.

Según la descripción hecha, los SE parecen ser prometedores para la sociología. El lenguaje simbólico y no numérico de los SE, la separación que efectúan entre conocimiento sustantivo y proceso/código del programa, la capacidad demostrada para el razonamiento dinámico, las exigencias de rigor lógico y de clarificación del pensamiento pueden facilitar un diálogo fructífero entre la IA y la sociología hasta ahora relativamente inédito. Con todo, se plantean algunos problemas en el uso de los SE por parte de la sociología, por ejemplo, en lo que se refiere al nivel de laxación o falta de precisión, o al menos de interpretación múltiple, que se da en muchas proposiciones y conceptos sociológicos y en la reactancia de algunas corrientes sociológicas a aceptar un cierto grado de formalización. De todas maneras, los sociólogos comienzan a darse cuenta de la importancia que, para la teoría y para la investigación social, tiene la IA, incluso se ha acuñado la denominación de origen IAS, inteligencia artificial social basada en SE, que consiste en la aplicación de las técnicas de la inteligencia mecánica y simbólica, en general las de la IA y, más concretamente, las de los SE a los fenómenos sociales.

La primera conferencia de sociología sobre IA se hace en 1984 (Gilbert y Heath, 1985), aunque la escasez y no excesiva consistencia de los trabajos no auguraba un buen comienzo. Además, el hecho de que, dentro de la sociología, los fenómenos más interesantes para la simulación como los de la interacción social se traten mayoritariamente dentro de concepciones algo reacias a la computación, no ha contribuido a que se dieran avances importantes en la IAS, al menos hasta la década de 1990. Tampoco favorece estas incursiones de la sociología en la simulación el hecho de que se dé una lacerante ausencia

óptimas para representar el conocimiento en forma jerárquica y como relaciones complejas causales.

7. Para soslayar esta dificultad se recurre a la estrategia de los sistemas pizarras, blackboard: los SE razonan por separado sobre la parte que tienen expertise y envían regularmente sus resultados actualizados a un sistema de datos participado, blackboard, que los examina (Brent, 1996). 
de la sociología y de los sociólogos en el núcleo duro que configura la ciencia cognitiva, salvo raras excepciones. Pero, como venimos insistiendo, surge, a partir de los noventa, un mayor interés dentro de la sociología por desarrollos como el de la inteligencia artificial distribuida (IAD) (Gasser, 1991); el de la teoría y tecnología de coordinación y cooperación; el de programas de lenguaje natural. Los especialistas en IAS han focalizado más su atención sobre la sicología que sobre la sociología, pero aunque la IA se ha centrado más en la modelación de la cognición individual, cada vez se ha ido incrementando más el interés por la inteligencia artificial distribuida (IAD); un campo en el que se tienen en cuenta las propiedades de la interacción ${ }^{8}$. En el mismo sentido que Piaget confiaba en el conocimiento social para comprender la acción individual, los investigadores en SE han comenzado a tratar el conocimiento y la acción social usando los resultados de la sociología del conocimiento. Hoy día muchos investigadores sociales centran su trabajo sobre los agentes situados en grupo y/o organizados, por ejemplo: los trabajos en áreas de la IAD, los estudios de agentes adaptativos complejos en estudios de la emergencia y cooperación, el rol de la estructura en la acción, etc. Con reticencias, se va estableciendo un intercambio, algo desigual y receloso aún por parte de los sociólogos, pero sin rubor ni complejos por parte de expertos en simulación, entre, por un lado, la sociología y, por otro, la ciencia de la simulación, por ejemplo: en análisis de textos y redes, evolución social, teoría de la organización, etc. (Carley, 1996).

Los SE han incidido de manera importante en el campo de la sociología, la teoría y la metodología. Su aplicación a la teoría sociológica ha tenido dos vertientes. La primera, en la teoría formalizada y deductiva. El creciente número de ensayos teóricos basados en las simulaciones de ordenador es una indicación de que los teóricos sociales ven nuevas posibilidades: la IA puede asegurar mejor la consistencia lógica de la teoría, es decir, asegurar si una teoría está bien formada y expresada sin redundancias, ayudar a descubrir nuevas implicaciones y a conectar hipótesis dispersas inspirando y evaluando nuevas teorías. Brent, Glazier y otros (1989) combinan la programación con reglas procedurales y marcos para elaborar y ampliar la teoría de Goffman, ERVING ${ }^{9}$. La segunda, en la teoría inducida a partir de los datos. Desde una vertiente más inductiva, la IA puede detectar pautas subyacentes basadas en el comportamiento observado y modelarlo en situaciones sociales (Carley, 1992; Carley y Nevwell, 1994;

8. El modelo IAD se estructura como agentes de comunicación múltiple, cada uno con un sistema de conocimiento complejo (Doran y Gilbert, 1994). Como SE, son de naturaleza simbólica. Las temáticas propias de la IAD se refieren a la relación entre agente y grupo, cognición y acción participada y coordinada, la planificación y el diseño organizativo (Carley, 1996).

9. La teoría de Parsons se ha tratado como un conjunto de reglas (Fararo y Skvoretz, 1984; Skvoretz y Fararo, 1995). Los SE se han usado también como modelos de razonamiento y examen de las decisiones organizativas (Masuk, 1990; Carley, 1996). Sylvan y Glassner (1985) ilustran el uso de la IA en 107 pasajes de Simmel (1955) a partir de 223 proposiciones formalizadas trasladadas a un programa inteligente (Benfer, Brent y Furbee, 1991). 
Bainbridge, Brent y otros, 1994) ${ }^{10}$. La aplicación de la IA a las metodológicas y técnicas de investigación ha crecido de manera considerable tanto en el área de los métodos cuantitativos ${ }^{11}$ como en la de los cualitativos $^{12}$.

\section{La inteligencia artificial social (IAS) en la nueva inteligencia artificial (NIA)}

En la última década se ha dedicado gran atención a las «máquinas de aprendizaje» con programas que aumentan el conocimiento y la habilidad procedural para aprender de la experiencia, en particular de los procesos de relación entre individuos y grupos en su adaptación a nuevos entornos. Cuando hablamos de NIA ${ }^{13}$ hablamos básicamente de redes neuronales artificiales (RNA) (Rumelhart y McClelland, 1987; Wasserman, 1993; Karayiannis y Venetsanopulos, 1993) y de algoritmos genéticos (AD) (Holland, 1975, 1992). La NIA puede contemplarse como una alternativa bottom-up a las aproximaciones top-down de los SE (Bainbridge, Brent y otros, 1994).

Aunque algunos de los trabajos sobre las RNA provienen de los años 50 esta aproximación fue eclipsada, de alguna manera, por las aproximaciones simbólicas de la IA clásica. Pero, a partir de mediados de los ochenta, las RNA han alcanzado éxitos incuestionables y notable popularidad. Las RNA son instrumentos que emulan el comportamiento de los sistemas nerviosos biológicos: la información se almacena en las conexiones ${ }^{14}$. Los nodos se ordenan o se establecen habitualmente en niveles secuenciales de tal manera que haya conexión entre nodos de niveles contiguos pero no entre los alternos ${ }^{15}$.

Los AG provienen de las teorías biológicas de la evolución. En ellos la población se adapta en el tiempo, dentro del proceso evolutivo de mutación

10. Brent (1986), Garson (1987), Schrodt (1989), Benfer y Furbee (1989) y Furbee (1989) usan SE inductivos para generar proposiciones teóricas a partir de datos. Glymour y otros (1987) desarrollan teorías que suponen modelos causales usando técnicas heurísticas (Benfer, Brent y Furbee, 1991).

11. Una revisión, en los 90, de los SE y otras técnicas de AI se pueden ver en Bainbridge, Brent y otros (1994), por ejemplo: programas de diseños de experimentos, como el DESIGNER RESEARCH $^{\mathrm{TM}}$; de muestreo como el EX-SAMPLE+ ${ }^{\mathrm{TM}}$; de elaboración de cuestionarios, como el MEASUREMENT \& SCALING STRATEGISTT ${ }^{\mathrm{TM}}$; de recogida de datos e información, como el DATA COLLECTION SELECTION ${ }^{\mathrm{TM}}$ (Brent, 1989); de reconocimiento de los métodos estadísticos apropiados para el análisis de datos, como el STATISTICAL NAVIGATOR $^{\mathrm{TM}}$ o el STATISTICAL \& SCALING STRATEGIST.

12. Se pueden plantear simulaciones próximas al interaccionismo, a temáticas culturales (Carley, 1996; Heise, 1992) y al lenguaje.

13. Algunos autores incluyen también los conjuntos borrosos, el caos y catástrofes y los simulated annealing.

14. Se dice conexionista porque supone que la inteligencia no consiste en la manipulación de símbolos, sino en las conexiones entre componentes.

15. Carley (1992) usa este modelo para examinar cómo la estructura organizativa constriñe la capacidad de las organizaciones aprovechando la experiencia aprendida por los agentes de la organización (Kontopoulos, 1993), para comprender la estructura social. Mi punto de vista es que las RNA son uno de los procedimientos de simulación más interesantes y fecundos en el futuro para la sociología. 
y reproducción, a un entorno en el que solamente sobreviven los más adaptados. Junto a las RNA me parece un modelo de simulación de largo alcance para la sociología, sobre todo en procesos evolucionarios (Freeman, 1993; Holand, 1992).

Ambos modelos de simulación, AG y RNA, han captado la imaginación de numerosos científicos sociales, sobre todo a partir de sus metáforas biológicoevolucionarias. Veamos algunas áreas de interés para su aplicación Los sistemas adaptativos complejos pueden simular agentes en su evolución social, para lo cual han de tener la dinámica propia de los sistemas no sólo adaptativos, sino también competitivos. Se trata de procesos en los que diversos agentes tratan de adaptarse al comportamiento de otros, de tal manera que producen propiedades globales en el sistema. Muchos trabajos sobre procesos evolucionarios se llevan a cabo por AG dirigidos por procesos de optimización de objetivos. Se puede simular, por ejemplo, la evolución de las redes sociales y los cambios organizativos. Los trabajos que se han realizado sobre redes sociales con estos modelos se han dedicado a poner de manifiesto las estructuras sociales y organizativas subyacentes de redes, otros se han centrado en estudiar cómo las estructuras específicas que condicionan el comportamiento de los agentes pueden hacerlos evolucionar adaptativamente: los trabajos de Carley (1991), Kaufer y Carley (1993) se refieren a las constricciones espaciales y sociales sobre la evolución del aprendizaje social. Los modelos de optimización y clasificatorios surgen para localizar soluciones, clasificar objetos usados, realizar particiones de grafos, importante para las redes sociales (Breiger, Boorman y Arabie, 1975; White, Boorman y Breiger, 1976) y para localizar particiones de equivalencia, por ejemplo, los AG en la partición de los miembros de un grupo en cliques o subgrupos basados en la proximidad entre individuos (Freeman, 1993; Carley, 1996).

\section{La simulación, la sociedad artificial y la sociedad real}

En este último apartado se repasan algunas cuestiones permanentemente importantes para la metodología sociológica y que inciden directamente en la simulación social.

¿Es adecuada y pertinente la representación social de los fenómenos o hechos sociales (agentes, intereses, interacción, estructura, etc.) implícita en los modelos de simulación a la concepción que de los hechos o fenómenos sociales se ha adquirido, más o menos consensuadamente, en sociología?

Los modelos de simulación social se han desarrollado originalmente a partir de los campos de la IA, la física, la biología, la evolución... Dado que el objeto y los procedimientos metodológicos de la sociología difieren de los de la ciencia natural, aunque puedan estar algo más próximos a los de la cognitiva, el trasvase a la sociología de modelos originados de las ciencias naturales puede ser problemático (Gilbert y Troitzsch, 1998). Eso no quita que la simulación 
esté revelándose eficaz y prometedora en lo social, como lo son y han sido los modelos matemáticos, que también provenían en buena medida de las ciencias naturales.

La primera pregunta que surge es si la imagen de la acción social, de los agentes, de la interacción, etc. y, en general, del sistema social implícito o explícito en el modelo social que se representa por la simulación es una representación pertinente, válida y parsimoniosa. Maturana y Varela (1987) definen un comportamiento/acción como una descripción que hace un observador (eliminaría lo de "como una descripción» que hace un observador) de un cambio o modificación que se da en un sistema con relación a un entorno con el que interactúan. El comportamiento es, pues, algo observable, pero para calificarlo como social será necesario que se lleve a cabo por agente(s), o tenga que ver con otro(s) agente(s) con un determinado propósito siempre que el otro agente, objeto del comportamiento, se considere también con propósito. El propósito puede consistir en ejercer una influencia en el comportamiento de otro (versión fuerte) o simplemente en tener en consideración el comportamiento de otro para decidir el propio (versión débil). Según mi entender, la condición para que un comportamiento sea social es que se lleve a cabo en vistas a o sea en sí (o proceda de) una interacción, lo que supone propósito.

¿Es necesario que los comportamientos o acciones lleven a la autorreproducción y al automantenimiento, definición de autopoiesis en Maturana y Varela (1987), para que sean sociales? En mi opinión, depende en qué sentido se entiendan ambas. Los propósitos, y en consecuencia los resultados de la acción, han de estar orientados a la producción y constitución (autoproducción), mantenimiento o conservación (autorreproducción), del Nosotros y de los Otros con los que los agentes se identifican o se distancian. Entendida así, la autopoiesis pasa a ser condición de lo social. El comportamiento de un sistema (agente, conjunto o estructura de agentes) es social si sus inter(acciones) o comportamientos lo son en dicho sentido (Gershenson, 2002). Parece que si partimos de un modelo que representa la sociedad (agentes, propósitos, interacción, estructura, etc.) como un conjunto de agentes o grupos (individuos o sujetos sociales) relativamente complejos que interactúan entre sí en un entorno que, en parte les determina y en parte contribuyen a construir (lo que supone tanto el reconocimiento de unas capacidades cognitivas como una dinámica y una distribución entre ellos de sus funciones y resultados de la interacción), algunas de las diferentes formas de simulación que hemos recorrido tienen características que parecen acoplarse bien a dicho modelo social y que superan las de otros modelos posibles. En este sentido, además de la pertinencia genérica social del modelo de simulación social, éste goza de otras virtualidades: su adecuación para el tratamiento de la incertidumbre, la versatilidad y el detalle de lo social, su adaptación a los procesos complejos, su capacidad descriptiva, explicativa y predictiva y al control de error y a la reproducción de un mundo social artificial (Seror, 1994). Pero se ha de insistir en el hecho de que los sistemas artificiales de simulación social no son sociales en sí mismos, dado que no son autopoiéticos en el sentido que he dado al término. Sí que 
son construcciones y mecanismos sociales, en el sentido de que su pertinencia social les viene de ser objetos, instrumentos, recursos y resultados de representaciones para la ejecución de mundos artificiales sociales. Es decir, que se ha de diferenciar entre la capacidad de producir lo social, que no es propio de la simulación, y su pertinencia social: los modelos de simulación social son, como todos los modelos, representaciones de comportamiento social que se leen y se ejecutan en programas y por ordenador, ni más ni menos. Mal servicio se hace a la simulación si se va más allá. Por el contrario, lo que surge de lo social son los sujetos sociales, sus interacciones, los cambios que producen o las estabilidades que se reproducen, los grupos y las estructuras, las emergencias que surgen... (Gershenson, 2002).

\section{Validación y sensibilidad}

La validación de la simulación tiene varias facetas. La primera es la validez interna, que asegura la coherencia interna entre el modelo de simulación y la representación de los hechos (o de la teoría) que se pretende simular. La validez de resultados es similar a la de los modelos metodológicos tradicionales y a la de la ciencia en general. Mide el grado de correspondencia entre los resultados simulados (esperados o predicciones) del modelo y los datos del mundo real. Pero para ello se ha de añadir, en simulación, la validez del proceso que asegura la correspondencia entre los mecanismos del modelo y los procesos que se dan en el mundo real; en esta validez se diferencia de los modelos matemáticos. En simulación no sólo se requiere que los resultados esperados por simulación se correspondan con los reales, validez de resultados, sino que se correspondan también los procesos, lo que no quiere decir que dichos procesos sean los mismos que se den en la realidad social: el diseño y funcionamiento con lógicas y procesos evolucionarios o con reglas o mecanismos de los AG, RNA o SE, sólo producen resultados simulares a los que operan con la mente. Una de las ventajas con relación a la validez habitual consiste en que los sistemas de simulación cuentan con programas que incluyen generadores de valores aleatorios, lo que asegura la ausencia de sesgo al comprobar su consistencia introduciendo diferentes datos y parámetros aleatoriamente en cada ejecución. Una vez que se ha validado el modelo con las condiciones iniciales y los valores de los parámetros, el análisis de sensibilidad permite conocer hasta qué punto el modelo está afectado por los supuestos que se han hecho, es decir, si el comportamiento es sensible a las variaciones de uno o más parámetros (Gilbert y Troitzsch, 1998).

\section{Explicación, descripción y predicción}

No se ha de confundir la precisión descriptiva (que es lo que se atribuye a veces a la simulación por ordenador) ni con la explicación teórica (que es la única que a veces se atribuye a los modelos matemáticos que formalizan teorías) ni con la predicción (que es la que a menudo se considera en los modelos metodoló- 
gicos). Aunque los modelos de simulación por ordenador permiten aumentar el realismo descriptivo, pueden tener también un valor predictivo y explicativo.

Los modelos de simulación, en tanto que representación de las características y procesos, por ejemplo, de las interacciones del mundo social, han de poseer reglas y/o procedimientos que puedan encontrar alguna equivalencia en la realidad social, así pueden facilitar la explicación teórica. De alguna manera, la explicación dada por la simulación pretende entrar más en la caja negra del proceso social. Los modelos predictivos contemplan los procesos como una caja negra sin tener en cuenta los posibles isomorfismos que el modelo de simulación puede guardar con la realidad; se centran en los resultados. Los modelos de simulación por ordenador pueden llenar en su diseño los dos objetivos: explicativos y predictivos. Hay científicos satisfechos con predicciones correctas (descripción precisa); otros están más interesados en aprender más sobre los mecanismos reales implicados (Taber y Timpone, 1996).

De todas formas, conviene insistir en que la conexión entre predicción y explicación no es directa ni evidente y ello aparece más claro en la simulación que en los modelos clásicos. La predicción puede ser buena aunque falle la explicación, pero el éxito de una teoría no es completo con sólo predecir satisfactoriamente unas observaciones. Por contra, explicaciones aparentemente completas en unos niveles no dan predicciones suficientes en otros. En las situaciones de complejidad, que es uno de los platos fuertes de la simulación social, podemos tener una explicación completa de los factores que afectan a la acción individual o de los agentes que no tiene por qué ser suficiente para la predicción de los comportamientos de un grupo o de una institución.

\section{Simulación y experimentación}

La simulación ostenta una gran similitud con la metodología experimental, aunque no sea lo mismo. Se comienza con un problema u objetivo que esté claramente definido y articulado, se elaboran y se proponen hipótesis, se testan y se evalúa el resultado. Todo ello antes de usar el ordenador. Las hipótesis surgen, como de costumbre, de la literatura, de la realidad o de ambas, ya que las simulaciones no se generan en el vacío. La naturaleza de las hipótesis es variada: predicción, descripción, explicación. El funcionamiento o puesta en marcha del programa dependerá y estará limitado por el diseño de la simulación y éste, a su vez, estará constreñido por las hipótesis. Pero el diseño requiere algo más, por ejemplo, el tener en cuenta las tareas, los tipos de lenguajes simbólicos o numéricos, la representación de los estímulos, los procesos elegidos (diferentes dentro de los SE), los diversos tipos de validación (Plunkett y Elman, 1997; Elman y otros, 1998). Un modelo de simulación se ejecuta muchas veces, variando las condiciones en las que funciona y se exploran los efectos de los diferentes parámetros.

Pero aunque la simulación tiene similitudes con la experimentación, no se trata de lo mismo, como tampoco lo es de la semiexperimentación que se aplica en sociología, aunque ésta tenga un ápice más de similitud con la simulación. 
La mayor diferencia consiste en que mientras en un experimento se controla el objeto real de interés, en una simulación se experimenta con un modelo y un mundo social artificial, no con el fenómeno en sí.

Los sistemas complejos, y en general los sociales, no resisten la descomposición o separación en sus partes o factores. Lo propio del planteamiento experimental es precisamente dicha disección: variar un factor mientras otro permanece constante para examinar sus efectos. Pero los sistemas sociales descansan en (y se crean a partir de) la interacción entre las partes; su comportamiento y propiedades emergen de dicha interacción que supera la mera adición de sus elementos. La llegada de la simulación por ordenador facilita el estudio de estos sistemas como totalidades, sin necesidad de reducir los fenómenos o sistemas sociales al estudio de sus partes.

La simulación hace, de alguna manera, cambiar la forma de hacer ciencia. Los «mundos artificiales», mundos subrogados de los reales, son el laboratorio y el experimento para la simulación. El cambio está en que ahora es posible explorar y manipular la información o un mundo artificial en lugar de la materia.

Para Galileo el control y la replicación del experimento eran parte inherente del método científico. Un sistema como el de Newton es simple, pues, aunque consista en un gran número de partículas o cuerpos que interactúan, lo hacen en base a una información puramente local de acuerdo con reglas rígidas; la física ha tenido la primacía en la experimentación clásica durante siglos consagrándose como la ciencia por excelencia. Por el contrario, en los sistemas complejos (red de tráfico en carreteras, mercado de stocks), los agentes, incluso en menor número, interactúan en función de información parcial, probable y no completa, son adaptativos e inteligentes, su comportamiento está influido por otros agentes y, aunque funcionen por reglas, están dispuestos y/o condicionados a cambiar dichas reglas de acuerdo con nuevas informaciones, adaptándose continuamente a su entorno para prolongar su propia supervivencia como sistema. La dificultad de los experimentos clásicos controlados y replicables es evidente en estos casos. La simulación por ordenador, como laboratorio experimental, se lleva a cabo sobre una estructura informativa de sistemas complejos. La ascendencia imparable de la IA, de la ciencia cognitiva, de los modelos evolucionarios, neurológicos, etc., no son más que la parte visible de un cambio fundamental, el de la materia y/o ondas que han sido sustituidas por la información del o de los sistemas que interactúan (Casti, 1999).

\section{Deducción, inducción y abducción}

Tanto la deducción como la inducción son el pan nuestro de cada día en la investigación, pero la simulación proporciona una tercera posibilidad. Partiendo de un conjunto de supuestos, se generan datos por procedimientos simulados que pueden ser considerados inductivamente a la hora de engendrar teorías ad hoc en un especie de intercambio entre estrategias, deductiva a veces y otras 
inductiva. La simulación es una manera de hacer ciencia en la interfase de los dos otros métodos estándar, el de inducción (búsqueda de pautas en la realidad), usado ampliamente en sociología, y el de la deducción (especificación de axiomas y sus consecuencias derivadas), por ejemplo, la teoría de juegos de la elección racional es un buen ejemplo deductivo. La simulación aparece como una "tercera vía». Similar a la deducción, puede comenzar con supuestos explícitos, pero, a diferencia de ella, no verifica teoremas; en su lugar genera datos que pueden analizarse inductivamente. A diferencia de la inducción, los datos simulados acostumbran a estar rigurosamente especificados por un conjunto de reglas u otros procedimientos, más que directamente extraídos del mundo real. Mientras la inducción se usa para encontrar pautas y la deducción para extraer consecuencias de los supuestos, los modelos de simulación pueden usarse para ayudar a la intuición. De alguna manera, la simulación es un experimento del pensamiento. Aunque los supuestos sean simples, las consecuencias pueden no ser obvias como puede pasar con los efectos emergentes; lo que no quiere decir que no haya propiedades emergentes que pueden ser deducidas formalmente. En los modelos económicos neoclásicos, en que los agentes racionales operan bajo poderosos supuestos de información disponible y la optimización puede distribuir los beneficios con mínimos costes. Pero si los agentes usan estrategias adaptativas en vez de optimización, la deducción de consecuencias es a menudo imposible, es el caso en que la simulación actúa como lámpara maravillosa (Axelrod, 1997).

El concepto de abducción de Peirce (1955) puede convenir bien a la metodología de simulación. La metodología implícita a la abducción es un encuentro a medio camino entre inducción y deducción, una especie de razonamiento retroalimentado, en el sentido de que se produce un cruce fructífero entre la observación sorprendente de los hechos o anomalías empíricas y la reflexión o razonamiento deductivo que proviene de la teoría. El acoplamiento es de adecuación, debido a que se encuentran razones para pensar que el modelo es verdadero. A esta conclusión se llega por un proceso continuo de ida y vuelta entre teoría y datos. De hecho, la mayor parte del razonamiento y de la práctica en la investigación sociológica tiene un indudable parangón con el razonamiento abductivo: unas ideas iniciales que se configuran en modelos simples, un recurso a los datos, una aproximación teórica de nuevo, etc.; es decir, un proceso de retroducción para ir conquistando la teoría por medio de modelos intermedios y construcción progresiva (Scheff, 1994).

\section{Bibliografía}

Axelrod, R. (1997). "Advancing the Art of Simulation in the Social Sciences Published». En Conte, C.; Hegselmann, R.; Terna, P. (eds.). Simulating Social Phenomena. Berlín: Springer, p. 21-40.

Bainbridge, W.S.; Brent, E.E.; Carley, K.M.; Heise, D.R.; Macy, M.W.; MArKovsky, B.; SKVOretZ, J. (1994). "Artificial Social Intelligence». Annual Review of Sociology, 20, p. 407-436. 
Benfer, R.A.; Brent, E.Jr.; Furbee, L. (1991). Expert Systems. Londres: Newbury Park, Nueva Delhi: Sage Publications.

BENFER, R.L.; FuRBEE, L. (1989). «Procustres analysis of Individual Configurations: Patterns and Axes Similarities». Journal of Quantitative Anthropology, 1 p. 65-80.

Breiger, R.L.; Boorman, S.A.; AraBiE, P. (1975). «An a Algorithm for Clustering relational Data with Applications to Social Networks Analysis and Comparison with Multidimensional Scaling». Journal of Mathematical Psychology, 12, p. 328-383. BRENT, E.E. (1986). «Knowledge-based Systems: A qualitative formalism». Qualitative Sociology, 9, p. 256-282.

- (1989). «Is there a Role for Artificial Intelligence in Sociological Theorizing?». En Blank, G.; McCartney, J.; Brent, E.E. (eds.). New Technology in Sociology: Practical Applications in Research and Work. New Brunswick, NJ: Transaction, p. 129-137.

- (1996). «Expert System and Their Role in Computational Sociology». Sociological Methods and Research, 25, 1, p. 31-59.

BRENT, E.E.; ThOMPSON, G.A. (1999). «Sociology: Modeling Social Interaction with Autonomous Agents». Social Science Computers Review, 17, 3, p. 313-322.

Brent, E.E.; Glazier, J.; JamtgaARD, K.; Wetzel, E.; Hall, P.; Dalecki, M.; BaH, A. (1989). «Erving: A Program to Teach Sociological Reasoning form the Dramaturgical Perspective». Teaching Sociology, 17, p. 38-48.

Brent, E.E.; Thompson, G.A.; VAle, W. (2000). «Sociology: A Computational Approach to Sociological Explanations». Social Science Computers Review, 18, 2, p. 223-235.

Brown, C. (1995). Chaos and Catastrophe Theories. Thousand Oaks: Sage Publications. CARLEY, K.M. (1988). «Formalizing the social expert's knowledge». Social Methodological Research, 17, p. 165-232.

- (1991). «A theory of group stability». American Sociological Review, 56, p. 331-354.

- (1992). "Organizational Learning and Personnel Turnover». Organization Science, 3, p. 20-46.

- (1996). "Artificial Intelligence Within Sociology». Sociological Methods \& Research, 25, p. 3-30.

Carley, K.M.; Newell, A. (1994). "The Nature of Social Agent». Journal of Mathematical Sociology, 19, p. 221-262.

CASTI, J.L. (1999). "The Computer as a Laboratory. Toward a theory of complex, adaptative system». Complexity, 4, 5, p. 12-14.

Castillo, E.; GutiérRez, J.M.; Hadi, A.S. (1997). Expert Systems and Probabilistic Network Models. Berlín: Springer.

CroOn, M.A.; vAN DE VIJVER, J.R. (1994). «Introduction». En CroOn, M.A.; VAN DE VIJVER, J.R. (ed.). Viability of Mathematical Models in the Social and Behavioral Sciences. Lisse Berwyn PA: Swets \& Zeitlinger B.V., p. 13-29.

DerecK, P. (1996). «Representation of Knowledge». En Boden, M.A. Artificial Intelligence. San Diego: Academic Press, cap. 3.

DiPreTE, T.A.; ForRistal, J.D. (1994). «Multilevel Models: Methods and Substances». Annual Review of Sociology, 20, p. 331-357.

DORAN, J.; GILBERT, N. (1994). «Simulating societies: an introduction». En GILBERT, N.; DORAN, J. Simulating societies. The computer simulation of social phenomena Londres: UCL Press University College London, cap. 1, p. 1-18.

Elman, J.L.; Bates, E.A.; Johnson, M.H.; Karmiloff-Smith, A.; Parisi, D.; PlunKeTT, K. (1998). Rethinking Innateness. Cambridge, Londres: A Bradford Book The MIT Press. 
FARARO, T.J.; SKVORETZ, J. (1984). «Institutions as production systems». Journal of Mathematical sociology, 10, p. 117-182.

FLIEDNER, D. (2001). «Six Levels of Complexity; A Typology of Processes and Systems». Journal of Artificial Societies and Social Simulation, 4, 1.

Forrester, J.W. (1969). Urban Dynamics. Cambridge Mass: MIT Press.

- (1971). World Dynamics. Cambridge Mass: MIT Press.

Fox, J. (1996). «Expert Systems and Theories of Knowledge». En Boden, M.A. Artificial Intelligence. San Diego: Academic Press, cap. 6, p. 157.

FreEMAN, L.C. (1993). «Finding Groups with a Simple Genetic Algorithm». Journal of Mathematical Sociology, 17, p. 227-241.

FreEMAN, L.C.; Douglas, W.R. (1993). «Using Galois Lattices to Represent Network Data». En MARSDEN, P.V. (ed.). Sociological Methodology. Oxford: Blackwell, p. 127-146.

FurbeE, L (1989). «A folk expert system: Soils classification in the Colca Valley, Peru». Anthropological Quarterly, 62, p. 83-102.

Garson, G.D. (1987). "Computer simulation in social sciences». Academic Microcomputing. Newbury Park: Sage, p. 110-138.

GASSER, L. (1991). "Social conceptions of knowledge and action: DAI foundations and open systems semantics». Artifcial Intelligence, 47, p. 107-138.

GERSHENSON, C. (2002). «Philosophical Ideas on the Simulation of Social Behavior». Journal of Artificial Societies and Social Simulation, 5, 3, p. 1-12.

GIERE, R.N. (1988). Explaining Science. Chicago: The University of Chicago Press.

- (1991). Understanding scientific reasoning. Fort Worth y Chicago: Holt, Rinehart and Winston Inc.

Gilbert, G.N.; Heath, C. (1986). «Text, competence and logic: An exercise». Qualitative Sociology, 9, p. 215-236.

- (eds.) (1985). Social Action and Artificial Intelligence. Aldershot England: Gower.

GILBERT, N.; CONTE, R. (eds.) (1995). Artificial societies :The computer simulation of social life. Londres: UCL Press.

Gilbert, N.; DORAN, J. (eds.) (1994). Simulation societies. The computer simulation of social phenomena. Londres: UCL Press University College London.

Gilbert, N.; Troitzsch, K.G. (1998). Simulation for the Social Scientist. Open University Press.

Glymour, C.; Scheines, R.; SPIRTes, P.; Kelly, K. (1987). Discovering Causal Structure. Nueva York: Academic Press.

GonZÁleZ, S., (2002). "Simulació i canvi social, una aproximació evolucionaria». Revista Catalana de Sociologia, 18, p. 89-102.

Griffiths, H.B.; OldKnOw, A. (1993). Mathematics of Models. Nueva York: Ellis Horwood.

Gross, D.; STrand, R. (2000). "Can Agent-Based Models Assist Decisions on LargeScale Practical Problems? A Philosophical Analysis. Use of Microstructurally Complex Models». Complexity, 5, 6, p. 26-33.

Guts, A.K.; Korobitsin, V.V.; LapteV, A.A.; Pautova, L.A.; Frolova, J.V. (2000). Social systems. Formalization and computer simulation: Text-book. Omsk: Omsk State University.

HeISE, D.R. (1992). "Computer assistance in qualitative sociology». Social Science Computer Review, 10, p. 531-543.

HEISE, D.R.; LEWIS, E.M. (1988). Introduction to ETHNO. Raleigh NC: National Collegiate Software Clearinghouse. 
Helbing, D. (1985). Quantitative Sociodynamics. Dordrecht: Kluwer Academic Publishers.

- (1994). «A mathematical model for behavioral changes by pair interactions and its relations to game theory». Angewandte Sozialforschung, 18(3), p. 117-132.

Holand, J.H. (1975). Adaptation in Natural and Artificial Systems. Ann Arbor Mich: University Michigan Press.

- (1992). «Genetic Algorithms». Science America, 267, p. 66-72.

Karayiannis, N.B.; Venetsanopulos, A.N. (1993). Artificial Neural Networks: Leaning Algorithms, Performance Evaluation and Applications. Boston: Kluwer. KaUfer, D.; CARLEY, K.M. (1993). Communication at a Distance: The Effect of Print on Socio-Cultural Organization and Change. Hillsdale NJ: Lawrence Erlbaum. KLÜVER, J.; SCHMIDT, K. (1999). «Topology, Metric and Dynamics of Social Systems». Journal of Artificial Societies and Social Simulation, 2, 3.

KONTOPOULOS, K.M. (1993). «Neural networks as a model of structure». The Logic of Social Structure. Nueva York: Cambrige University Press, p. 243-267.

LeIK, R.K.; MeeKer, B.F. (1975). Mathematical Sociology. Nueva Jersey: Prentice Hall Englewood Cliffs.

- (1995). «Computer simulation for exploring theories: Models of Interpersonal Cooperation and Competition». Sociological Perspectives, 38, 4, p. 463-482.

MACY, M.W. (1991). «Learning to cooperate: Stochastic and tacit collusion in social exchange». American Journal of Sociology, 97, p. 808-843.

MASUK, M. (ed.) (1990). Organization, Management and Expert Systems: Models of Automated Reasoning. Berlín y Nueva York: Water de Gruyter.

Maturana, H.R.; VARELA, F.J. (1987). The Tree of Knowledge: The Biological Roots of Human Understanding. Boston y Londres: Shambala.

MolenaAr, I.W. (1994). "Why Do We Need Statistical Models in the Social and Behavioral Sciences?». En CRONN, M.A.; VAN DE VIJVER FOnS J.R. (ed.). Viability of Mathematical Models in the Social and Behavioral Sciences. Lisse Berwyn PA: Swets \& Zeitlinger B.V., p. 101-112.

Peirce, C.S. (1955). «Abduction and Induction». En Buchler, J. (ed.). Philosofical Wrintings of Peirce. Nueva York: Dover.

PlunKetT, K.; Elman, J.L. (1997). Exercices in Rethinking Innateness. Handbook for Connectionist. Simulations. Cambridge y Londres: A Bradford Book The MIT Press.

PYKA, A. (2001). «Special Issue on Applied Simulation Analysis». Journal of Artificial Societies and Social Simulation, 4, 3.

Rumelhart, D.E.; McClelland, J.L. (1987). Parallel Distributed Processing. Cambridge: MIT Press.

Saaty, T.L.; Joyce, A.M. (1981). Thinking With Models. Oxford: Pergamon Press. SANDOR, J. (1989). «Investigation of Agricultural Soils at Lari, Colca Valley, Peru». En Guillet, D. (ed.). Cognitive and Behavioral Studies of Soil Management in Peru. Report to the National Science Foundation.

SCHEFF, T.J. (1994). Microsociology. Discourse, Emotion and Social Structure. Chicago y Londres: The University of Chicago Press.

SCHRODT, P.A. (1989). "Artificial intelligence and formal models of international behavior». American Sociologist, 19, p. 71-85.

- (1991). «Prediction of interstate conflict outcomes using a neural network». Social Science Computer Review, 9, p. 359-380.

SEROR, A. C. (1994). «Simulation of complex organizational process: a review of methods and their epistemological foundations». En GIlbERT, N.; DORAN, J. (eds.). 
Simulation societies. The computer simulation of social phenomena. Londres: UCL Press University College London, cap. 2, p. 19-40.

SHubiK, M. (1991). Theorie des Jeux et Sciences Sociales. París: Economica.

Simmel, G. (1955). Conflict and the Web of Group Affiliation. Nueva York: Free Press. SKVORETZ, J.; FARARO, T.J. (1995). «The evolution of systems of social interaction». Current Perspectives in Social Theory, 15, p. 275-299.

- (1996). «Generating Symbolic Interaction. Production Systems Models». Sociological Methods and Research, 25, 1, p. 60-102.

Sylvan, D.; Glassner, B. (1985). A Rationalist Methodology for the Social Sciences. Oxford UK: Basil Blackwell.

TABER, C.S.; TimpOnE, R.J. (1996). Computational Modeling. Londres y Nueva Delhi: Tousand Ooks y Sage University.

Thom, R. (1975). Structural Stability and Morphogenesis. MA: Bejamin Reading. Walliser, B. (1977). Systèmes et modèles. París: Editions du Seuil.

Wasserman, P.D. (1993). Advanced Methods in Neural Computing. Nueva York: Van Nostrand.

WeIDlich, W.; HAAG, G. (1988). Interregional Migrations. Berlín: Springer.

White, H.C.; Boorman, S.A.; BreIGER, R.L. (1976). «Social Structure from Multiple Networks: I. Blockmodels of Roles and Positions». American Journal of Sociology, 81, p. $730-780$. 